\title{
Traffic Aware Strategic Aircrew Requests (TASAR)
}

\author{
Mark G. Ballin ${ }^{1}$ and David J. Wing ${ }^{2}$ \\ NASA Langley Research Center, Hampton, VA, 23681
}

\begin{abstract}
Under Instrument Flight Rules, pilots are not permitted to make changes to their approved trajectory without first receiving permission from Air Traffic Control (ATC). Referred to as "user requests," trajectory change requests from aircrews are often denied or deferred by controllers because they have awareness of traffic and airspace constraints not currently available to flight crews. With the introduction of Automatic Dependent Surveillance-Broadcast (ADS-B) and other information services, a rich traffic, weather, and airspace information environment is becoming available on the flight deck. Automation developed by NASA uses this information to aid flight crews in the identification and formulation of optimal conflict-free trajectory requests. The concept of Traffic Aware Strategic Aircrew Requests (TASAR) combines ADS-B and airborne automation to enable user-optimal in-flight trajectory replanning and to increase the likelihood of ATC approval for the resulting trajectory change request. TASAR may improve flight efficiency or other user-desired attributes of the flight while not impacting and potentially benefiting the air traffic controller. This paper describes the TASAR concept of operations, its enabling automation technology which is currently under development, and NASA's plans for concept assessment and maturation.
\end{abstract}

\section{Introduction and Background}

A ircraft operating in the National Airspace System under Instrument Flight Rules (IFR) generally must fly trajectories approved by Air Traffic Control (ATC). The approved trajectory flown by an IFR aircraft is the trajectory originally specified in the flight plan or subsequent ATC clearance received prior to takeoff, modified by changes issued or negotiated and approved by ATC after takeoff and throughout the flight. The approved trajectory often does not coincide with the aircraft operator's most efficient or preferred trajectory. Less-desired trajectories can be the result of non-optimal routes, altitude restrictions, and/or speed restrictions issued by ATC before or during the flight, or of changing conditions or priorities during the flight. Some causes of in-flight priority changes are unanticipated weather convection or turbulence development, the need to make up time as a result of an earlier reroute to avoid traffic or weather, the need to delay arrival due to fleet operator constraints or traffic congestion at the destination, and the need to increase altitude as fuel is burned to improve efficiency. As a result, pilots occasionally have a need or desire to change their trajectory while in flight. The desired change may be a revised lateral route, a climb or descent to a different altitude, a change in airspeed, or a combination. It may be of a temporary nature, such as a heading change to avoid weather, or a long-term nature, such as a diversion to an alternate airport.

Because ATC has responsibility to separate IFR aircraft, it maintains authority over the trajectories of all IFR aircraft in controlled airspace, and IFR pilots are not permitted to make changes to their approved trajectory without first receiving permission from ATC. The operational procedure to request a trajectory change is for the pilot to prepare the request and, when appropriate, communicate it to the air traffic controller. The controller will assess the request with respect to nearby traffic and other factors and issue an approval, an amendment, a deferral, or a denial. The pilot then proceeds as instructed.

Referred to as "user requests," trajectory change requests from aircrews may not be living up to their full potential to provide user benefits. Traffic information is currently not available to most flight crews, and consequently, a trajectory change request has a reasonable chance of not being approvable by the controller because

1 Project Scientist, NASA NextGen Concepts and Technology Development Project, Mail Stop 156A, mark.g.ballin@nasa.gov, Associate Fellow, AIAA

2 ATM Research Engineer, Mail Stop 152, david.wing@nasa.gov, AIAA Member

1

American Institute of Aeronautics and Astronautics 
of resulting conflicts. Disapproved user requests are an operational detriment to everyone involved. They cost workload for the pilot and controller, contribute to radio frequency congestion, and do not produce a more desirable trajectory. In addition, conflict-free opportunities for improving the trajectory can remain undiscovered by pilots because of the lack of onboard traffic information and automation to compute trajectory changes that are more optimal.

Automatic Dependent Surveillance - Broadcast (ADS-B) has been established as a surveillance infrastructure that will provide substantial benefits to both airspace users and air navigation service providers in the future. The FAA has mandated that all aircraft operating in current Mode-C airspace be equipped with ADS-B transmit capability ("ADS-B Out") by 2020. System benefits increase with the number of aircraft equipped, so there is interest in increasing ADS-B equipage levels as quickly as possible, preferably long before the mandate takes effect. Therefore, near-term applications are sought that provide user benefits large enough to justify the cost of equipping aircraft with ADS-B capability. This paper summarizes one such ADS-B application under investigation.

\section{TASAR Overview}

ADS-B provides an opportunity for airspace users to gain more utility from their trajectory change requests. Aircraft that equip with ADS-B receivers ("ADS-B In") gain access to the key information - timely and accurate traffic surveillance - needed to formulate trajectory change requests that avoid other aircraft and therefore are more likely approvable by ATC. Flight deck automation $1^{, 2}$ developed by NASA for advanced self-separation concepts of operation 34 provide trajectory probing computations that can also be applied in current operations to aid in the identification and formulation of user-optimal, conflict-free trajectory requests. The concept of Traffic Aware Strategic Aircrew Requests (TASAR) combines ADS-B and flight deck automation for in-flight decision-aiding and replanning to increase the likelihood of ATC approval of pilots' trajectory change requests, improving the efficiency or other user-desired attribute of the flight, while not impacting and potentially benefiting the air traffic controller. In addition to ADS-B surveillance, TASAR can leverage ground-based information services via data link or internet access, as well as on-board weather radar, to identify weather hazards to be avoided and other conditions affecting flight optimization and ATC approval.

\section{A. Potential User and ATC Benefits}

Aircraft operators benefit when they are able to make timely modifications to their aircraft's trajectory during flight to improve upon a less-than-optimal condition. Each operator may have unique optimization criteria, specified for each flight to meet the needs of the operator's business model. For some, saving flight time is paramount, whereas for others, minimizing fuel burn or maintaining comfortable ride quality is the priority. Others yet may have more complex optimization requirements, such as reducing overflight fees (for operations in other countries where this applies) or extending range to avoid a fuel stop delay. TASAR provides flexibility for diverse operators to make different adjustments according to their particular needs. It also provides automated monitoring to detect improvement opportunities that flight crews and dispatchers may otherwise miss.

Having flight optimization decision-making capability onboard the aircraft benefits the user in several ways. The capability can be customized to that particular aircraft's type and performance. It will have direct access to avionics for the active navigation trajectory and actual aircraft state (e.g., accurately computed current weight), as well as direct access to information about the local environment (e.g., sensed winds and weather). It also provides "on call" availability of flight-optimization support to the pilot, allowing better workload management. Onboard application also allows the user to tailor the settings to meet the individual business needs for that flight, including optimization criteria and threshold of improvement warranting the pilot's attention. Since no new capabilities are required on the ground to enable or support TASAR, individual users gain access to immediate benefits for each aircraft they equip, without dependence on ground-service implementation schedules. As a "per aircraft" capability, TASAR provides benefits independent of other users equipping their aircraft for TASAR. A companion paper provides an initial estimate of potential user benefits of TASAR.5

TASAR may also benefit ATC in reducing unproductive use of the voice frequency and controller workload associated with user requests by reducing the number of requests that are unapprovable due to traffic conflicts. If other ATC constraints are also taken into account by TASAR, benefits to ATC may increase further. An example is for TASAR to discourage user requests while the aircraft is estimated to be near handoff, when requests are typically deferred to the downstream sector controller. Reduction of unapprovable user requests frees the controller to be more efficient, which in turn benefits the ATC system and all airspace users. 


\section{B. Potential System Benefits}

Through timely in-flight replanning, TASAR facilitates an agile response to uncertainties inherent in operation within the National Airspace System, providing the potential for reducing impacts of uncertainty by increasing control authority and response rather than relying solely on improved predictions of future system state. In addition, TASAR may provide two important benefits in meeting the challenge of airspace system modernization. Its nearterm operational use will provide critical information leading to the design and development of advanced airspace system concepts that involve a high degree of aircraft and flight crew autonomy. Perhaps more importantly, TASAR may provide a user incentive for equipping aircraft with ADS-B, thereby accelerating equipage well in advance of the mandate's 2020 deadline.

\section{A Unique Approach}

To achieve these benefits, a unique approach to the TASAR design and the strategy for its implementation is proposed. The approach consists of three interdependent elements: a new flight deck function that directly benefits the airspace user, a design requiring minimal certification and operational approval, and a collaborative and sharedcost NASA/user exploration and development of the capability.

First, TASAR is designed to provide value to the airspace user. The concept offers a new capability to the flight crew: the ability to continuously optimize the aircraft's flight plan. Moreover, TASAR provides the capability to do this in the typical air carrier environment of traffic in proximity, airspace restrictions, and traffic flow management constraints. Traditional approaches to take advantage of surveillance afforded by ADS-B have focused on the cockpit display of traffic information. There have been few near-term ADS-B applications that make use of automation to support crew decisions, and they use automation only to provide simple functions. TASAR embodies the opposite approach: it makes use of automation to provide an optimization function, while the display of traffic information to the pilot is of little importance. Similar ground-based approaches to flight plan optimization may also provide benefits, but the TASAR approach allows tailoring of optimization criteria to the specific objectives of each flight. It also takes advantage of the trajectory generation capabilities designed for the aircraft and uses real-time information specific to each aircraft's unique situation. Because the TASAR concept is aircraft-centric, all automation and pilot procedures are fully dedicated to a single aircraft, resulting in timely responses to changing situations. Furthermore, as a "per aircraft" application that does not require other participating aircraft to be viable, TASAR has the potential to provide immediate benefits for each aircraft that is equipped, an important and powerful incentive to entice early participation and therefore more widespread adoption of ADS-B.

Second, TASAR is designed to require minimal certification and operational approval. TASAR imposes no required changes on controller procedures, equipment, or training. It does not provide a safety-critical function, nor does it affect the safety of the flight. Pilots would use TASAR to make a more informed trajectory change request to ATC, but the safety of this trajectory change remains entirely with ATC. TASAR provides no new authorization for the pilot to act without ATC approval. Since no operational credit is expected, TASAR will not result in the aircraft flying trajectories it would not otherwise already be authorized to fly. TASAR involves no automation that flies the aircraft. Rather, the existing aircraft systems under control of the pilot would follow the new trajectory. TASAR also does not rely on the display of ownship position or traffic to the pilot on a map. The software application that supports TASAR is designed to be classified as Electronic Flight Bag (EFB) Type B software. FAA Advisory Circular 120-76B6 currently prohibits a moving map display with ownship position for Type B software. Type B software may be hosted on any EFB hardware class. While it does require FAA Flight Standards District Office approval and Aircraft Evaluation Group evaluation, it does not require FAA Aircraft Certification Service design approval. Type B software does not require compliance with RTCA DO-178B, "Software Considerations in Airborne Systems and Equipment Certification." These factors facilitate an expeditious design, development, and authorization cycle for TASAR technology and approval for operational use.

These two elements enable the third and potentially most critical element: a collaborative and shared-cost exploration and development of the capability between NASA and interested airspace users. It may be possible for NASA to provide the basic TASAR software application to the public domain at no cost. If the user provides the necessary airborne surveillance capability and application hosting hardware, the NASA software application enables the user to achieve benefits from their investment. The user would employ the TASAR system in normal operations and provide feedback to NASA with the purpose of making the software more useful. To the extent possible, NASA would implement changes and release updated versions of the application. This cyclic enhancement strategy will depend on rapid software updates and hence minimal software certification requirements, but if successful, it is expected to lead to a relationship where both NASA and the users are co-innovators. The users will discover new ways to use the capability and new benefits of airborne surveillance, thereby adding incentives for voluntary ADS-B equipage. 


\section{Concept Description}

The purpose of TASAR is to advise the pilot of possible trajectory changes that would be beneficial to the flight and increase the likelihood of ATC approval of pilot-initiated trajectory change requests, thereby increasing the portion of the flight flown on or near a desired trajectory. Examples of desired trajectories include the most fuel efficient trajectory, a minimum flight time trajectory, a trajectory that meets a desired arrival time, and the lowest turbulence trajectory. Traffic surveillance information on nearby aircraft is received by the TASAR-equipped aircraft, referred to herein as the "ownship." An onboard software application (the "Traffic Aware Planner" or TAP) processes the surveillance information and performs conflict probing of possible changes to the ownship trajectory, either pilot-entered or automatically computed. In addition to surveillance information, the TAP application also may process other data as available from onboard sensors and databases or from data links. Information important to in-flight replanning may include convective weather regions, turbulence regions, terrain, wind field predictions, restricted airspace, and sector boundaries. Using ownship aircraft performance data and pilot preferences, the TAP application computes fuel burn or other desired trajectory attributes, and trajectory request optimization may be performed to minimize or maximize a pilot-specified attribute while avoiding traffic conflicts.

Information pertinent to the operation is indicated to the pilot on a cockpit interface. Such information may include the results of the conflict probe of a pilot-entered trajectory change, a recommended trajectory change modification that avoids nearby known traffic, a trajectory-change opportunity (or several alternatives or ranges of maneuvers) automatically identified by the TAP application, the incremental time and fuel saved or expended relative to the current trajectory, and other desired attributes of the trajectory change. The pilot uses this information in the decision of whether to make a trajectory change request of ATC and what request to make. Figure 1 shows an example interface screen for TAP in the Auto Mode (discussed in the next section), indicating several trajectory improvement opportunities available to the pilot.

The TAP application may provide the function of constraining trajectory change opportunities to named waypoints or other means to facilitate voice communication of the request. This aspect of the innovation allows its use in airspace, aircraft, or time frames where data link is not available for transmitting trajectory change requests to ATC. Normal pilot procedures are then used to issue the request, and normal ATC procedures are used to approve or disapprove the request. TASAR imposes no required changes on controller procedures, equipment, or training.

\section{A. Modes of Operation}

Two operational modes for the TAP application are defined: automated monitoring for opportunities (Auto Mode) and pilot initiated use (Manual Mode). In Auto Mode, TASAR is a passive capability that requires no ongoing or repetitive pilot action. The TAP application automatically performs a continuous assessment of opportunities for improving the performance of the flight according to any such goals and parameters specified by the pilot prior to or during the flight. A typical goal would be to maximize fuel efficiency, and the pilot could specify, for instance, a threshold of minimum improvement (e.g., $100 \mathrm{lbs}$ of fuel saved) required to qualify as an identified opportunity worth bringing to the pilot's attention. In addition, the trajectory change must not conflict with known traffic. Other considerations in the optimization search include avoiding known hazards, such as weather detected onboard or uplinked from a ground service, known

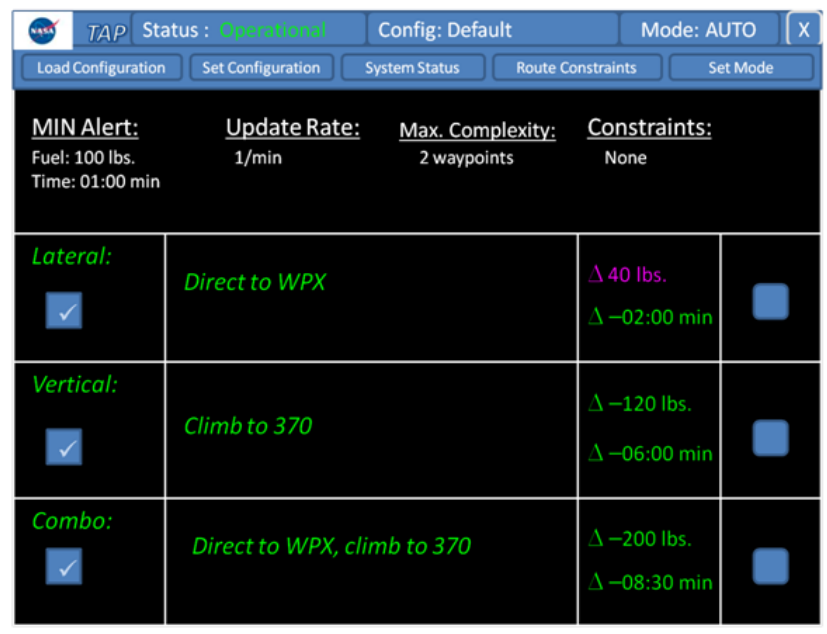

Figure 1. Example user interface for TAP in Auto Mode, indicating results of automatic monitoring for trajectory change improvement opportunities. turbulence, and terrain. Other ATC constraints, such as proximity to sector boundaries, may also be taken into account. The TAP application produces recommendations to the pilot that simultaneously consider the optimization goal, the traffic, the hazards and other constraints, and the ownship performance capabilities and limitations. The TAP application could be configured to produce a single optimal recommendation, a set of several alternatives, or potentially a continuous range of maneuvers. A list of available trajectory change options based on the pilot's pre-entered preferences is continuously available to the pilot for immediate use. 
In the Manual Mode, the pilot makes manual use of TASAR capability when a trajectory change is desired or when such a need is anticipated and the pilot wants to be prepared with a request at the opportune moment. Figure 2 shows an example user interface for TAP in Manual Mode. The pilot enters a desired trajectory change into the TAP application interface. Alternatively, the TAP application (if sufficiently integrated with onboard systems) could be designed to directly sense trajectory modifications entered by the flight crew into the Flight Management System (FMS) or other normal flight control interfaces. The entered or sensed trajectory change is probed by the TAP application for potential traffic conflicts. In addition, computations are made on the attributes of the change, for instance fuel burn saved or expended. The results of the probe and computations are provided to the pilot. If the probe indicates a conflicted route, the TAP application also

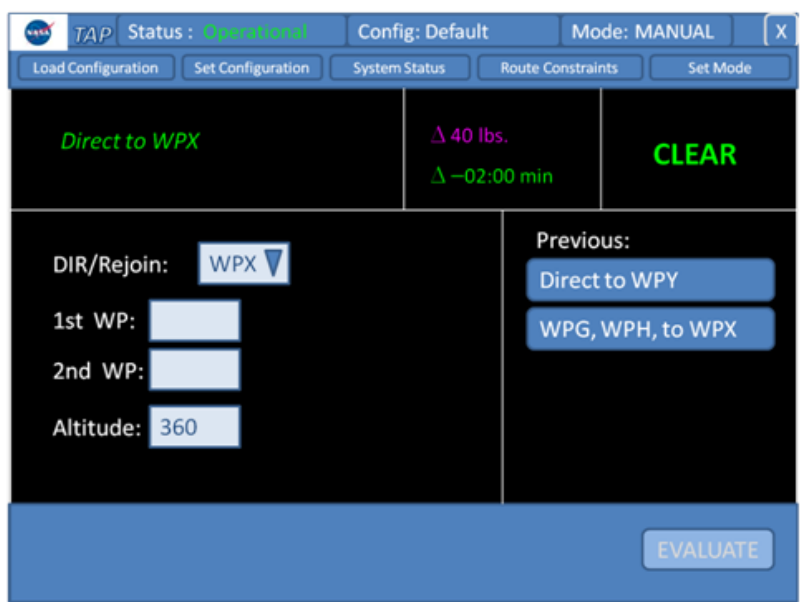

Figure 2. Example user interface for TAP in Manual Mode, indicating evaluation results of a trajectory change entered by the pilot.

provides a mechanism to compute a conflict-free modification to the desired trajectory change. Manual Mode may also be used by the flight crew to evaluate trajectory change proposals provided by ATC in response to the crew's request. For this use, conflict probing within TASAR may need to be temporarily disabled.

\section{B. Pilot Procedures}

Pilot use of TASAR is completely optional. TAP application information is advisory only, and the pilot remains authorized to make ATC requests without TASAR.

In Auto Mode, pilot procedures are mostly passive. The pilot activates the TAP application in this mode for any segment of the flight that opportunity advisories are desired, although TASAR is primarily intended for use above 10,000 feet. For those flight segments where advisories are not desired, such as times when the pilot determines no requests will be made (e.g., frequency too congested), the pilot deactivates this mode of the TAP application. Prior to activation, or at any time after activation, the pilot sets or updates the user preference settings. For example, the pilot may change the criteria for route optimization from best fuel efficiency to best time to destination. During the flight, while Auto Mode is active, the TAP application periodically probes for trajectory opportunities. When one or more opportunities are identified that meet or exceed the threshold criteria, the TAP application notifies the pilot and displays the recommend trajectory change(s) and supplemental information to aid the pilot's decision (e.g., estimated flight time saved). It displays verbiage to communicate the request expeditiously to ATC and, depending on the degree of avionics integration, provides the option to load the request into a data link message.

In Manual Mode, pilot procedures for TASAR involve data entry to the TAP application interface. During the flight, when the pilot identifies a need or desire to make a trajectory change request to ATC, the pilot uses the TAP application to probe the desired change prior to making the request. The pilot enters the desired trajectory change either directly through the TAP application interface or through existing flight control systems of the aircraft that are monitored by the TAP application. The TAP application probes the desired change for traffic conflicts and displays the results of the probe to the pilot. If a conflict is predicted on the desired trajectory change, the TAP application computes and displays one or more alternative modifications that meet user preference criteria. It displays verbiage to communicate the request expeditiously to ATC and, depending on the degree of avionics integration, provides the option to load the request into a data link message.

Once the preceding procedures are complete, the pilot then uses normal procedures (voice or data link) to make the trajectory change request, including determining the appropriate time to make the request. The pilot is not obligated to make the request as specified by the TAP application, nor to make any request at all. The information from the TAP application is advisory only. When a request to ATC is made, no reference to the TAP application or TASAR capability is required, since no special consideration by ATC is being requested. The pilot proceeds as instructed by ATC's response. 


\section{Example Scenario}

An example of TASAR concept use is shown in Figure 3. In the figure, the TAP-equipped aircraft (Aircraft A) was on schedule prior to movement of convective weather into its planned flight path. The aircraft was rerouted around the weather, and the extended path (shown as "initial route") resulted in a delay in its predicted arrival over its next waypoint (Fix B). The weather is slowly clearing out of the way, and the crew of Aircraft A would like to make up as much of this lost time as possible. There is traffic in proximity, and crossing traffic (Aircraft C) constrains a simple direct-to-fix replanning solution. The flight crew therefore uses TAP to compute a conflict-free, weatheravoiding, path that saves flight time. The new path (shown as "new route") also saves fuel, but is optimized to save time based on TAP's pilot optimization preference inputs. The pilot receives a display of an ATC voice request from TAP in written words as the pilot would speak them. If the pilot chooses to make the request to ATC, the controller may then grant it or reject it.

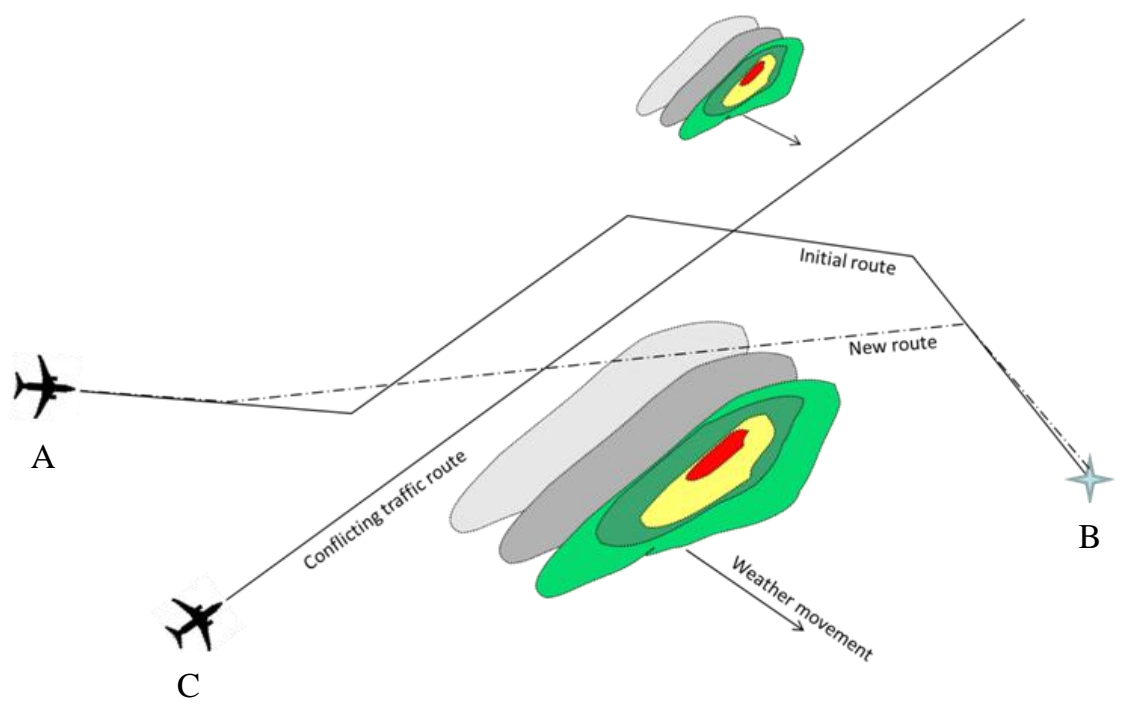

Figure 3. Example of using TASAR to save flight time.

\section{The Traffic Aware Planner}

The TAP application is a flight crew decision support tool designed to support the TASAR concept. It will most likely be hosted on a Class 2 EFB in near-term implementations. TAP makes use of algorithms developed for the Autonomous Operations Planner (AOP), ${ }^{1,2}$ which is a research application developed by NASA for exploration of advanced self-separation concepts such as the Autonomous Flight Rules. ${ }^{3,4}$ The NASA research that led to AOP was founded with two assumptions. First, self-separating aircraft will need to be integrated in the same airspace with aircraft that rely on ground-based separation services. Second, the two airborne functions of traffic deconfliction and conformance to airspace and flow management constraints will need to be solved simultaneously to achieve acceptable performance. These assumptions resulted in the development of AOP's primary function: an in-flight strategic replanning capability that accounts for traffic. AOP contains an automated trajectory optimizing function that accounts for all known traffic, airspace constraints (such as convective weather regions to be avoided), and externally imposed flow management constraints such as a required time of arrival at a fix. AOP makes use of traffic aircraft intent information, if it is available, to reduce false alerts and increase trajectory efficiency. Using the trajectory generation function of the aircraft's FMS, AOP generates an optimal conflict-free trajectory based on the pilot's optimization preferences. TAP makes extensive use of this same capability to support the TASAR concept. AOP also incorporates functions to support the AFR concept's maneuver rule that does not permit the intentional creation of new traffic conflicts as a result of a maneuver. These "conflict prevention" functions are also used by TAP to support pilot "what-if" planning.

\section{A. TAP Functions}

Functions planned for inclusion in the initial release of the TAP application include:

- Traffic trajectories processing: Creates a four-dimensional trajectory prediction for each known traffic aircraft.

- Ownship trajectory processing: Creates a four-dimensional trajectory from the ownship's state and intent. Flight mode segments are generated using the aircraft's flight plan, guidance modes, autoflight settings, and a performance model of the aircraft. 
- Hazard data processing: Builds and maintains a model of the hazard areas that will be accounted for by the trajectory optimization function. Hazard areas are static or dynamic regions of airspace that the aircraft must avoid or the pilot prefers to avoid.

- Procedural constraint processing: Maintains and applies a database of constraints associated with current ATC procedures for approving trajectories. The constraints are used by the TAP application functions that produce trajectories to ensure that the trajectory change requests do not run counter to standard procedures used by ATC.

- Pilot trajectory change input function: Monitors pilot input devices and produces a trajectory prediction corresponding to a proposed change in flight plan, flight mode, or autoflight setting.

- Conflict probing: Determines whether a specified ownship trajectory will conflict with any traffic trajectories or hazard areas. The trajectories of ownship and traffic may include buffering methods to account for uncertainties in trajectory prediction along specific segments or for extra distance desired to be maintained from traffic or hazards. Buffers are anticipated to be sized based on what is found to be acceptable to the air traffic controller through testing. A conflict is indicated if the ownship (or its buffered location) is predicted to come within a specified lateral/vertical volume of any traffic aircraft (or its buffered location) or breach the boundary of any hazard area. The function may be used with a single ownship trajectory specified by the pilot, multiple trajectories specified by the trajectory optimization function, or a "sweep" of trajectory changes defining a range of acceptable requests.

- Trajectory optimization function for Auto Mode: Computes one or more trajectory changes, if available, that improve desired attributes (such as fuel efficiency) of the flight. It searches through a wide range of possible modifications to the current trajectory, including different lateral route patterns, cruise altitudes, and combined lateral/vertical maneuvers. Both immediate and delayed maneuvers are considered. It takes into account trajectory constraints, including traffic, hazard areas, and crossing restrictions, returning only solutions that meet these constraints and provide sufficient buffering as specified by user settings. It computes desired attributes of the trajectory (such as fuel burn) and seeks the optimal value of these attributes while remaining conflict-free. It may optimize multiple objectives through a weighted fitness function.

- Trajectory change resolution function for Manual Mode: Produces a modified trajectory based on the pilotspecified preferred trajectory that remains clear of traffic and hazards and is unlikely to be seen by ATC as producing a conflict. In searching for resolution alternatives, the function may consider one or more degrees of freedom, such as vertical modifications to the desired altitude or climb/descent rate and lateral modifications to the desired track angle or route. It computes desired attributes of the trajectory (such as fuel burn) and seeks the optimal value of these attributes while remaining conflict-free. It may optimize multiple objectives through a weighted fitness function. Variants of this function may exist, each corresponding to strategic flight modes (i.e., flight-plan based) and tactical flight modes (i.e., pilot direct control of the autoflight system guidance targets).

- Voice request conversion: Adjusts the trajectory change request to use named waypoints or other means to facilitate voice communication of the request to ATC. Using a database of defined waypoints, navigation aids, or other shorthand conventions, the function seeks the nearest such points that can be used to approximate the optimized or pilot-selected trajectory change with minimal loss to the trajectory's desired attributes. The function rechecks the trajectory for conflicts and only returns trajectories that meet the original requirements.

- Pilot preference interface: Allows the pilot to specify and update the user preferences by which the TAP application operates while in flight. Examples of preferences include the desired trajectory attributes for optimization (e.g., fuel efficiency), the thresholds of improvement that warrant notifying the pilot, the rate at which automated optimization is attempted, the buffers to be applied around traffic and hazard areas, and the fidelity of voice request conversion to be performed.

- Proposed trajectory change pilot interface: Allows the pilot to specify trajectory changes to be probed by the TAP application. The mechanism may be a dedicated TASAR input device or, depending on the degree of avionics integration, may be the automated sensing of the FMS or other normal flight control interfaces. It supports pilot modifications to the flight plan and cruise altitude. Future versions may support tactical flight mode settings (heading, track, speed, and vertical speed).

- Trajectory change acceptability indicator: Indicates to the pilot the results of the conflict probe, i.e., whether a pilot-specified trajectory change is sufficiently free of conflict that a trajectory change request could be made to ATC and likely be approved. In future versions, the indicator may also show "at a glance" a range of maneuvers considered likely to be acceptable. 
- Opportunity available indictor: Indicates to the pilot the availability of one or more trajectory optimization solutions that the pilot may consider. The indicator may include the estimated improvement in one or more desired attributes of the trajectory (e.g., fuel saved). Depending on the degree of avionics integration, the mechanism may include the ability to load the trajectory change request in a data link message for transmission to ATC and/or to load it in the FMS for execution.

- ATC request verbiage indicator: Displays to the pilot the output of the voice request conversion function, i.e., the key verbiage (e.g., "direct to WPX, climb to 370") that facilitates ATC requests made over the voice channel.

\section{B. TAP Hosting Options}

Several TAP hosting options are possible, and each may have utility depending on the specific operational needs of the various airspace users. Options include Class 1, 2, or 3 electronic flight bags and full integration into the aircraft's avionics. In its simplest form, TAP could be hosted on a Class $1 \mathrm{EFB}$, a stand-alone platform not integrated with aircraft systems (other than power). ADS-B data and ownship position can be communicated to the device over a wireless network, and additional information from ground sources such as Next-Generation Radar (Nexrad) weather data and supplemental traffic information may similarly be acquired using airborne internet access. The minimal certification level of Class 1 EFBs makes this the lowest cost option to gain TASAR capability. However, the benefits will likely be minimal given the lack of access to onboard avionics systems and data.

Implementation of TASAR with a Class 2 EFB permits the TAP application to have direct, read-only access to data from the aircraft's systems, such as the FMS and flight control interfaces. The tool's access to aircraft system data, such as the flight plan, would significantly enhance its ability to optimize the route of flight. Class 2 EFBs are also generally an economic choice, given their relatively low certification level.

The TAP application may also be hosted on a Class $3 \mathrm{EFB}$, or equivalently, the TAP application capabilities can be fully integrated with the aircraft's avionics. The advantage of full integration is the ability to send trajectory solutions generated by the TAP application to the aircraft system for data link to ATC, the ability to load the solutions directly into the FMS, and the placement of solutions on forward-field-of-view displays. This connection to aircraft systems would significantly increase TASAR benefits by making available to the pilot a far more diverse and flexible set of trajectory change options. These may include complex reroutes, multi-step altitude changes, and combinations of these maneuvers. Class 3 and full integration hosting options may also make displays of ownship and traffic on a moving-map display a viable option. Given the high certification level of integrated avionics, including Class 3 EFBs, this option would likely be the most expensive.

\section{Ongoing Research and Development}

NASA is currently conducting research and development of the TASAR concept. Areas of applicability and utility to the airspace users are under study, and a TAP Version 1 prototype is in development. Expected opportunities for use and benefits have been quantified and are the subject of the companion paper. ${ }^{5}$ Requirements for equipment certification and operational approval are also being investigated, including a determination of suitability for each class of EFB as a hosting platform. A pilot assessment of the TAP human interface design will be performed using human-in-the-loop simulation and flight trials. To achieve acceptable rates of user request approval from ATC, a study of impacts on controllers and the use of supplemental surveillance and other information are also planned for investigation. Design and development issues include: the modification of mature AOP functions for the TAP application and refactoring code as necessary for instantiation as EFB Type B software; additional customization of the TAP optimization function based on user-specified criteria; modification of the optimization trajectory patterns to facilitate voice requests; and design and development of the flight crew interface. Later versions of TAP may include the use of 4D predicted wind fields, convective weather provided by XM or airborne internet sources fused with on-board radar, the use of a data link to communicate user requests to air traffic controllers, and the inclusion of additional airspace and ATC procedure databases needed by the procedural constraint processing function to improve acceptance rates of the user requests.

\section{Conclusion}

TASAR is a concept of operation that leverages ADS-B surveillance to provide direct user benefits. It is designed to be achievable in the near term and may provide immediate benefits for those who choose to equip. Each single aircraft receives benefit from equipping without relying on other aircraft to equip with ADS-B In. No new ground-based services or infrastructure are required, and it should have minimal certification and operational 
approval requirements. Over time, the ADS-B surveillance environment is expected to increase, thereby providing additional utility through a more complete picture of traffic aircraft to the airborne trajectory optimization capability. It is proposed that a NASA/user co-innovation partnership strategy be utilized. NASA would provide the TASARenabling software application, and the airspace user would provide the required on-board equipage, informal evaluations from voluntary use in normal operations, and feedback to NASA, including ideas for additional functions that would provide additional benefits. NASA would then use this feedback to develop and release application updates.

\section{References}

1. Ballin, M. G., Sharma, V., Vivona, R. A., Johnson, E. J., and Ramiscal, E., "A Flight Deck Decision Support Tool for Autonomous Airborne Operations," AIAA Paper No. 2002-4554, August, 2002.

2. Karr, David A.; Vivona, Robert A, Roscoe, David A.; DePascale, Stephen M.; and Wing, David J..: "A Flexible Platform for Research in Flight-Deck Support for Airborne Self-Separation" 12th AIAA Aviation Technology, Integration, and Operations (ATIO) Conference, Indianapolis, IN, September 2012.

3. Wing, David J. and Cotton, William B., "For Spacious Skies: Self-Separation with 'Autonomous Flight Rules' in US Domestic Airspace," AIAA-2011-6865, 11th AIAA Aviation Technology, Integration, and Operations (ATIO) Conference, Virginia Beach, VA, September 2011.

4. Wing, David J. and Cotton, William B., "Autonomous Flight Rules: A Concept for Self-Separation in U.S. Domestic Airspace," NASA TP-2011-217174, November 2011.

5. Henderson, Jeffery M.; Idris, Husni; and Wing, David J., "Preliminary Benefits Assessment of Traffic Aware Strategic Aircrew Requests (TASAR)," 12th AIAA Aviation Technology, Integration, and Operations (ATIO) Conference, Indianapolis, IN, September 2012.

6. "Guidelines for the Certification, Airworthiness, and Operational Approval of Electronic Flight Bag Computing Devices," Advisory Circular 120-76B, Federal Aviation Administration, June 1, 2012. 